\title{
Panorama eines Umbruchs
}

\author{
Die Modernisierung der Mathematik im Rückblick
}

\author{
Norbert Schappacher
}

Jeremy Gray, 2008: Plato's Ghost. The Modernist Transformation of Mathematics. Princeton: Princeton University Press, geb., $515 \mathrm{~S}$., $46,95 €$, ISBN-13: 978-0-691-13610-3.

Unbestreitbar veränderte sich die Mathematik im Übergang vom 19. zum 20. Jahrhundert grundsätzlich: Der Auftritt nicht-euklidischer Geometrien; die Geburt der Mengenlehre als grundlegende, allerdings antinomiengeplagte Disziplin; eine runderneuerte formale Logik; das Aufkommen der algebraischen Strukturen (wie beispielsweise Gruppe und Körper) als neuartige, abstrakte Objekte der Algebra; die Flexibilisierung des Raumbegriffs für die moderne Topologie ebenso wie für Funktionenräume, welche Ozeane von Funktionen zu einer Struktur vereinten, wo früher vertraute Exemplare individuell studiert wurden - diese und einige andere historische Phänomene erwecken den Wunsch nach einer einheitlichen Erzählung. Herbert Mehrtens hat eine solche 1990 in seinem Buch Moderne - Sprache - Mathematik als Erster geliefert. Dabei beschränkte er sich bewusst auf die reine Mathematik und deren Entwicklung vorwiegend in Deutschland. Sein Kernbegriff war die Moderne, die ihm einerseits übergreifender Epochenbegriff war und andererseits zur Benennung der in dieser Epoche sich bekämpfenden Lager diente: modern versus gegenmodern, letzteres mitunter zur radikalen Antimoderne gesteigert, etwa in völkischen Ideologien zum mathematischen Schaffen. Als modern bezeichnete Mehrtens dabei solche mathematischen Konzepte, die von außermathematischen Gegebenheiten unabhängig gedacht wurden. Moderne mathematische Theorien arbeiten autonom an einem formalen System. David Hilberts paradiesische Freiheit des modernen Mathematikers und sein Postulat der prinzipiellen Lösbarkeit aller mathematischen Probleme (gegebenenfalls nach geeigneter Kontextverschiebung) beruhten auf dem Verzicht, den mathematischen Grundbegriffen (wie Punkt, Gerade, Ebene im Falle der Geometrie) von vornherein referentielle Bedeutungen beizulegen. 
Weiter setzte Mehrtens seinen mathematikspezifischen Modernitätsbegriff in kulturelle und politische Verortungen ein - wozu Konstellationen wie der Streit des liberalen Hilbert mit dem reaktionären Gottlob Frege ja auch verleiten können.

Jeremy Grays gewaltiges Buch lässt sich nicht zuletzt als eine Wiederaufnahme der Mehrtens'schen Arbeit nach anderthalb Jahrzehnten divergenter Debatten lesen (vergleiche dazu den Abschnitt I.1.2). Allerdings setzt sich Gray deutlich von Mehrtens' Ansatz ab, dessen „post-marxistischen Geist“ (S. 10) er ebenso ablehnt wie den „nebulösen“ Begriff der Moderne (modernity, S. 12 f.) als umfassendes Epochenmerkmal. Sein Ziel ist es vielmehr, die modernist transformation der Mathematik zwischen 1890 und 1930 (S. 1) in einer möglichst großen Vielfalt mathematischer Disziplinen - Ausnahmen sind in Abschnitt I.1.3.2 erläutert - ebenso zu verfolgen wie in benachbarten Bereichen der Philosophie und Psychologie, bis hin zur Populärliteratur, und das alles in transnationaler Perspektive.

Was diese modernist transformation der Mathematik für ihn ist, definiert Gray gleich zu Beginn in einem einzigen Satz Kleist'scher Prägung (Gray nennt es eine brisk definition):

Here, modernism is defined as an autonomous body of ideas, having little or no outward reference, placing considerable emphasis on formal aspects of the work and maintaining a complicated - indeed anxious - rather than a naïve relationship with the day-to-day world, which is the de facto view of a coherent group of people, such as a professional or discipline-based group that has a high sense of seriousness and value of what it is trying to achieve. (S. 1)

Mit dem hier eingerückten Wort „anxious“ setzt er einen eigenen Akzent, der sich an kulturhistorische Analysen des frühen 20. Jahrhunderts anlehnen kann. ${ }^{1}$ Schon 2004 hatte Gray in einem Artikel in Science in Context für anxiety als mathematikgeschichtliche Kategorie geworben. In der Mitte des Buches (S. 266-277) kommt er darauf zurück, beginnend mit einer willkommenen, knappen Bilanz der Entwicklung des 19. Jahrhunderts und ihrer Folgen für den Begriff des mathematischen Beweises. Bei Mehrtens wirkten mindestens die Vertreter der modernen, fortschrittlichen Richtung unverzagt. Gray hingegen präsentiert eine Handvoll von Autoren, bei denen er anxiety ausmacht: Charles Hermite, Edwin Bidwell Wilson, Leopold Kronecker, Federigo Enriques, sowie Oskar Perron in seiner Tübinger Antrittsvorlesung von 1911. Wie Gray selbst sagt (S. 274), handelt es sich bei den älteren Autoren um ausgesprochene Vertreter von Mehrtens' Gegenmoderne ${ }^{2}$, welche schwerlich unter die obige Definition fällt. Deren mutmaßliche anxiety könnte als Reaktion auf Effekte des Modernismus gedeutet werden; Gray geht jedoch möglichen Parallelen zur Modernitätskritik von Bergson bis Heidegger nicht nach und beschränkt übrigens das Phänomen der anxiety auf die Zeit vor dem Ersten Weltkrieg. Grays Diagnose beruht meines Erachtens auf heiklen Deutungen. Kommt in der auf den Seiten 272 und 273 diskutierten Passage aus Kroneckers 
letzter öffentlicher Vorlesung von 1891 wirklich die „classic language of anxiety" zum Ausdruck? Und dass Gray Perrons Betrübnis über den damaligen Zustand der Mengenlehre als anxiety auffasst, kontrastiert mit Perrons forscher Persönlichkeit und dem übrigen Tenor der Antrittsvorlesung, die auf mich eher wie der zupackende Zwischenbericht des Poliers von der Großbaustelle wirkt.

Methodisch kaum einfacher sind Probleme der Periodisierung. Kunstgeschichtlich kommt die Moderne wohl erstmals in Giorgio Vasaris Vite Mitte des 16. Jahrhunderts zum Ausdruck. Literaturgeschichtlich markiert die Querelle des anciens et des modernes das Ende des 17. Jahrhunderts als Neuanfang. Christophe Charle argumentiert 2011 in seiner Discordance des temps, dass als natürlicher Periodenbeginn für seine überwiegend auf Frankreich zentrierte Studie nicht die französische Revolution, sondern das Jahr 1830 anzusehen sei. Damit sind wir schon fast am Anfang des von Mehrtens betrachteten Intervalls der Mathematikgeschichte angelangt, das andererseits bis zum Einsetzen der Postmoderne reicht und somit, wenn auch als „Fortsetzungsgeschichten", den Nationalsozialismus ebenso behandelt wie das Projekt Bourbaki und die Arbeiten Turings. Auch für Leo Corrys Studie zur modernen Algebra (Corry [1996] 2004) ist es wesentlich, die Reflexion auf algebraische Strukturen bei Bourbaki und in der Kategorientheorie mitzuberücksichtigen. Gray bezieht zwar das ganze 19. Jahrhundert als status quo ante des Umbruchs in seine Darstellung ein; sein zweites Kapitel, Before Modernism, beginnt er mit den ersten Jahren der École polytechnique, Gaspard Monge und der projektiven Geometrie im 19. Jahrhundert. Aber im 20. Jahrhundert beschränkt er sich im Wesentlichen auf die Zeit bis 1930. So lässt er im siebten Kapitel, After the War, die Herausforderungen des Hilbert'schen Optimismus, von Brouwers Intuitionismus über Gödels Unvollständigkeitssatz bis zur modernen Berechenbarkeitstheorie, Revue passieren und betont die frühe Reife der formalen Logik und ihre Bedeutung für die Philosophie der Mathematik. Damit gelangt Gray an das Ende seiner Darstellung. Dieser Haltepunkt in der Mitte zwischen den Weltkriegen verdiente es, genauer reflektiert zu werden. Wie zum Beispiel artikuliert sich Bourbakis Projekt, das erst in der Mitte der 1930er Jahre allmählich entsteht, zwischen Tradition und moderner Erneuerung? Ist eine solche Zäsur in der Mitte des „einundreißigjährigen Weltkriegs“ 1914-1945 (Hobsbawm) bei der angewandten Mathematik erkennbar? Derartige Fragen scheinen mir mehr Aufschluss zu versprechen als Grays allgemeine Bemerkungen über die Wellenbewegung zwischen highly structured und densely structured Spielarten der Mathematik (S. 453).

Nach dem schon erwähnten zweiten Kapitel, das mit der Beschreibung eines circa 1880 konstatierbaren traditionellen Konsenses endet, wird die eigentliche Transformation in den beiden folgenden Kapiteln Mathematical 
Modernism Arrives und Modernism Avowed ausgerollt. Gray bietet hier wie im Großteil des Buches - vor allem eine dichte Folge von kurzen Essays über Autoren, Texte oder Episoden seiner Geschichte. Die Darstellung erinnert insgesamt an ein Panorama in der ursprünglichen Verwendung des Begriffs, die man heute nur noch selten besichtigen kann (etwa das Bourbaki-Panorama in Luzern): ein runder Pavillon, dessen innere Wand in fortlaufenden Tableaus mit vielen Einzelheiten eine denkwürdige Geschichte illustriert. Grays Essays sind die Frucht von Jahren eingehender Beschäftigung mit dem betrachteten Zeitraum und der einschlägigen Sekundärliteratur. Dass seine Darstellung gleichzeitig auf Formeln und mathematisches Detail zugunsten möglichst breiter Verständlichkeit verzichtet - vier Sätze der projektiven Geometrie und ein kleines Glossar mit Fachterminologie im Anhang dienen demselben Zweck -, sollte auch der Allgemeinbildung im Reden über diese Zeit zugute kommen. Die Kehrseite des gebotenen Reichtums ist, dass - trotz zahlreicher Querverweise zwischen den Abschnitten und trotz übersichtlicher Unterscheidung verschiedener Typen der Modernisierung nach den großen mathematischen Disziplinen Geometrie, Analysis, Algebra - die vielen berücksichtigten Einzelpersonen und Ereignisse einer einheitlichen Erzählung der modernist transformation im Wege stehen. Es gibt nicht einmal ein detailliertes Inhaltsverzeichnis, in dem man die Folge der mit drei Ziffern nummerierten Abschnitte überblicken könnte. Nicht nur lassen sich, was wenig verwunderlich ist, Akteure häufig kaum nach der Mehrtens'schen Dichotomie modern - gegenmodern einordnen. Die Lektüre des Buches wirft besonders angesichts weniger bekannter Autoren die Frage auf, wie der Flickenteppich individueller Agenden zur Epochengeschichte steht. Soll die Geschichte der Moderne in lokale Modern(ism)en aufgelöst werden - von denen dann freilich zu fragen wäre, inwiefern jede(r) von ihnen diese Bezeichnung verdient? Oder sollten vielmehr primär einzelne Forscher oder Autorengruppen mikrohistorisch untersucht werden, wobei die Frage nach ihrem Verhältnis zu Tendenzen der Moderne nur ein Erkenntnis fördernder Gesichtspunkt unter anderen sein könnte?

Wilhelm Wundt als Akteur nicht zu übergehen, rechnet sich Gray als besonderes Verdienst an (S. 4), und auch dem außerhalb Italiens eher vergessenen Federigo Enriques widmet er sich an mehreren Stellen. Seine Darstellungen aber (insbesondere auf den Seiten 393-398 und 356-365) beschränken sich auch in diesen beiden Fällen schon aus Platzgründen auf Einführungen, und diese produktiven Autoren bleiben dem Leser vor allem als Farbflecke in einem bunten Epochenbild in Erinnerung. In dem Abschnitt 4.5: Modernist Objects (S. 235-239) wendet sich Gray zuerst den von Kurt Hensel eingeführten $p$-adischen Zahlen zu. Deren extravagante Rolle in Bezug auf übliche Maßbegriffe für Brüche macht sie in der Tat provokant modern. Mathematikhistorisch besonders interessant ist aber, wie Hensel - den sonst 
nichts dazu prädestinierte - im Laufe der Jahre zahlentheoretischer Arbeit in der Nachfolge Kroneckers dazu kam, ein Vorzeigeobjekt der Moderne zu kreieren (vgl. Petri 2011). Moritz Epple ist in seiner Entstehung der Knotentheorie 1999 analogen Fragen im Einzelnen nachgegangen, worauf Gray auch verweist (S. 237).

$\mathrm{Zu}$ den Stärken von Grays Darstellung gehört die Behandlung von mathematikbezogenen Themen, die selten in die Mathematikgeschichte integriert werden. So zeichnet er nicht nur die verschiedenen philosophischen und psychologischen Debatten nach, sondern bietet im fünften Kapitel auch eine vergleichende Skizze mathematikhistorischer Aktivitäten im späten 19. Jahrhundert in Deutschland, Frankreich und Italien an sowie im sechsten Kapitel hoch willkommene Abschnitte über künstliche internationale Sprachen.

Insgesamt bietet das Buch also keine fertige Bilanz. Aber es wird ein unvergesslicher, vielfältig geschürzter Knoten im Taschentuch eines jedes Historikers bleiben, der über dieselbe Zeit arbeitet.

\section{Anmerkungen}

1 Neben dem von Gray auf S. 267 zitierten Buch Burrows könnte man heute beispielsweise auch auf den Abschnitt Une modernité malheureuse (S. 335 f.) in Charle 2011 verweisen.

$2 \mathrm{Zu}$ Hermite, der gewisse Tendenzen explizit als „modern“ ablehnte und ihnen sein konservativ-ganzheitliches Bild der Mathematik entgegenstellte, vergleiche die reichen Zitate und ihre Analyse von Goldstein 2011.

\section{Literatur}

Charle, Christophe, 2011. Discordance des temps. Une brève histoire de la modernité. Paris: A. Colin.

Corry, Leo, [1996] 2004. Modern Algebra and the Rise of Mathematical Structures. 2. Aufl. Basel u. a.: Birkhäuser.

Epple, Moritz, 1999. Die Entstehung der Knotentheorie. Kontexte und Konstruktionen einer modernen mathematischen Theorie. Braunschweig/Wiesbaden: Vieweg.

Goldstein, Catherine, 2011: Un arithméticien contre l'arithmétisation: les principes de Charles Hermite. In: Dominique Flament und Philippe Nabonnand, Hg., Justifier en mathématiques. Paris: Editions MSH, 129-165.

Gray, Jeremy, 2004. Anxiety and Abstraction in Nineteenth-Century Mathematics, Science in Context, 17, 23-47.

Mehrtens, Herbert, 1990. Moderne - Sprache - Mathematik. Eine Geschichte des Streits um die Grundlagen der Disziplin und des Subjekts formaler Systeme. Frankfurt a. M.: Suhrkamp.

Petri, Birgit, 2011. Perioden, Elementarteiler, Transzendenz - Kurt Hensels Weg zu den p-adischen Zahlen. München: Dr. Hut. 
Norbert Schappacher

Institut de Recherche Mathématique Avancée

Université de Strasbourg

7 , rue René Descartes

67084 Strasbourg Cedex

France

E-Mail: schappacher@math.unistra.fr 